\title{
Calibration Techniques of Analog to Digital Converters(ADCs)
}

\author{
Chakradhar Adupa, Rajesh Kumar Srivastava, Sreenivasa Rao Ijjada
}

\begin{abstract}
Data converters play a vital role in converting analog signal information to its equivalent digital information. In general purpose computing devices, industrial electronic equipment, and biomedical equipment especially in display device modules, they make use of these converters for displaying accurate results very effectively with high precision. these data Converters establish a key rolein between the analog transducers sensors, actuators to digital signal processing and helps for easy data processing in systems, like wireless communication systems and also in multi-channel biomedical devices etc.,There are variety of converters are available for different applications at different levels considering their parameters such as speed of conversion resolution, gain accuracy, etc., and In this paper, a comprehensive overview of the errors and the different calibration are discussed. The research work will give the different concepts and techniques for error calibration of $A D C$ for an effective output with low INL and DNL obtaining high ENOB.
\end{abstract}

Keywords- ADC, Calibration, Errors, INL, DNL, ENOB.

\section{INTRODUCTION}

Data converters are present in every electronic application majorly the analog to digital converters are becoming platform for humans to give commands to the electronic devices. There are lot of applications related to data converters but their accuracy is the concern for their usage etc.,. The basic requirement of designing $\mathrm{ADC}$ is to have more accuracy in terms of INL, DNL and ENOB. There are several error sources in ADC which degrades the critical factors and to subdue the errors we look into calibration.

Digital calibration techniques [1]-[10],found in literature states that there are lot of advancements are done in designing of pipelined ADC but even though also found so many drawbacks are facing due to the analog signal paths during the calibration which will be performed in foreground or background[1]-[4].

The forefront calibration method stands from the absence of capability of tracking, and these methods are subtle to temperature and voltage variations and the aging of the device. In Analog paths, the interference cannot be escaped. In traditional methods, Calibration from LSB to MSB is done by accurate boot strapping algorithm, lower stages calibrated accommodated in the circuit and continued till the end stage finished. During the calibration process, the

Revised Manuscript Received on September 14, 2019.

ChakradharAdupa, IEEE Member: Asst Professor, Dept. of ECE,SR Engineering College,Warangal,Telangana, INDIA. (E-mail: adupa.chakradhar@gmail.com)

Rajesh Kumar Srivastava, SCI. /ENGR. "SD” VLSI Design Division SCL-Chandigarh, INDIA. (E-mail: rajesh@scl.gov.in)

SreenivasaRao Ijjada, IEEE Member, Asst Professor, Dept. of ECE, GITAM University, Visakhapatnam, Andhra Pradesh, INDIA. (E-mail: srinivasanaidu.ijjada@gitam.edu) in most critical applications such as medical, space, defense

implementation of sequential operations are very much complex in practice, since they need analog switch circuits in pipeline structures. This entire mechanism makes complicate the digital control logic and adds analog circuitry overhead. This will results more power and delays the conversion process as in the literature in [1]-[8].

\section{SOURCES OF ERRORS}

The ADC's specifications has been deviated and limited mainly due to both linear and non linear errors presented in the data converters. The sources of these errors are the zero and full scale voltage offsets, Differential and Integral nonlinearities, Signal noise ratios, error in quantization process, Effective number of bits and spurious free dynamic range.

\section{Significance of these errors:}

Offset is the fluctuation seen in the ADC's characteristics between the actual and exact characteristics. this offset is observed in the converters because of the offset which is caused by the comparators which usually used in converters.

1. Offset errors creates a limitation in the ADC functionality. The substantial positive offset error causes the output value to steep at maximum before the input voltage reaches topmost level.and a substantial negative offset error gives a zero output value to the compact input voltages

2. Gain error is a change in the slope of the stair case in ideal to actual characteristics. It primarily gathers the error to considerable for higher output codes. in general the un calibrated voltage reference gives rise to gain error and the output code will scale with the voltage reference, hence the different voltage references will gives us different output codes.

3. INL error is the fluctuation seen in the LSB of a real transfer function from a straight line. and DNL is the fluctuation seen in the real step width to its absolute of 1 LSB. these are the two kinds of ways in which the errors can be found in ADC's functionality.

4.The error which occurs in analog to digital conversion process where it out-turn from its impotence to responds to small changes of less than a magnitude.

These errors can be reduced by calibration. Several calibrations schemes are discussed below.

\section{LITERATURE ADDRESSING THE ISSUES}

Myung Jun Choe and Bang Sup Song and Kantilal Bacrania [11] have implemented a pipelined and background offset trimming method to 13 bit data converter 
circuit which converts from analog to digital information at a rate of 40 mega samples per second. the mentioned cmos converter background offset trimming is planned with highly Over sampling DS modulator which intensifies the resolution of "Folders" after 12 bits. Here The framework offset trim circuit constantly computes and fine tunes offset of turn up amplifier without butt into the usual operation of the circuit. in the process of framework offset trimming a part of pipelined ADC with additional circuitry is considered for misconception computation. The folding amplifiers are denoted as TA(Turn up amplifier) so TU2,TU3, and TU4 means three amplifiers are seen in the figure and each of these amplifiers are composed of three differential current controllers.

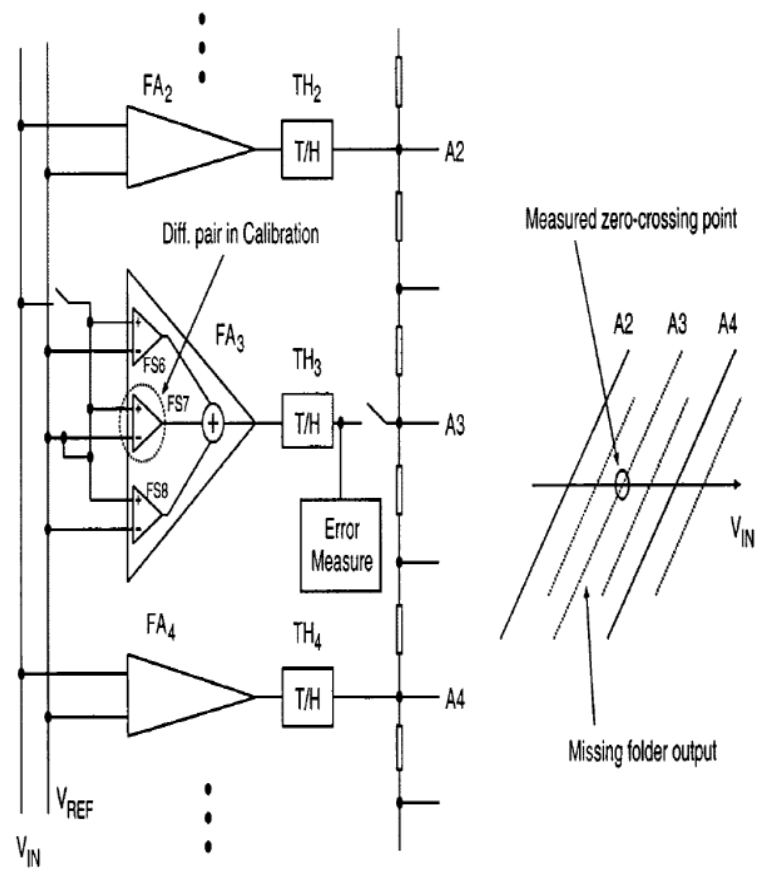

Fig.1 Backdrop offset Measurement

In figure 2 the detailed discussion regarding the offset computing and calibration is made. where both the things can be done independently. here highly over sampled converters are used to measure the output of the FA and at the same time the measured deviations are stored in memory. After every new measurement the up down blocks updates them selves. the arrangement is done for each current switch such that there will be a proper adjustment will be done by using a dissimilar voltage on a twin capacitors which needs a regular recharge.

The results shows that a SFDR of 82 decibels at 40 mega samples per second and the measured INL as 5 LSB and DNL is around +/-2LSB separately. and this work is done at 0.5 um cmos technology which shows that it consumes $800 \mathrm{~W}$ of power at $5 \mathrm{v} \mathrm{dc}$. and this method of implementation also proves that the feasibility of availing interpolation turn up ADC for elevated resolution at gaint speed is seen by joining the planning of two intensification and framework trimming idea.

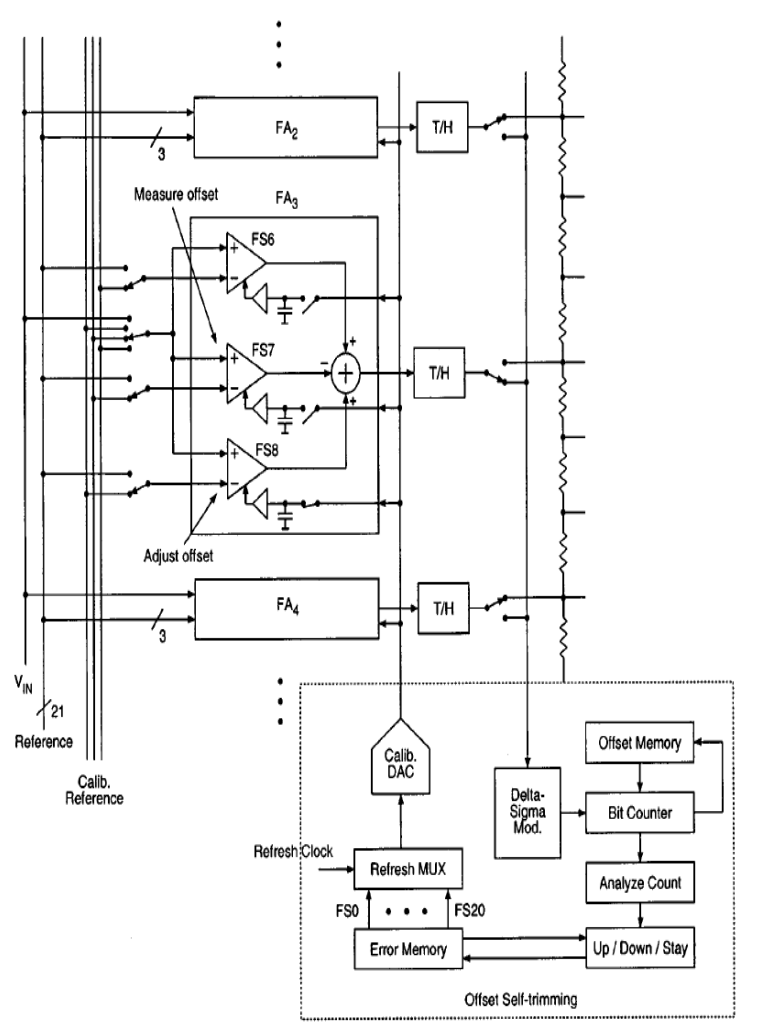

Fig.2 Backdrop offset trimming

Yun Chiu, Cheongyuen W. Tsang, BorivojeNikolicand Paul R. Gray [12] presents an adaptive digital technique which is closely to pipeline ADCs for calibration. in conventional approach the linearity is achieved by adjusting the analog component values but in this scheme it concludes component misconception from altering outcome and appeals digital post processing to precise those results. the method which is suggested at this point is making a immediate analogy to the channel equalization problem generally found in electronic Communications.

In the new architecture which is proposed in pipelined ADC where the component errors collected from entire stages of the pipeline are pull out at the same time using an Flexible FIR digital Filters it is similar to the twisting in communication channel. here the analog signal is path is thoroughly intact so the greatest conversion speed is allowed by fixed device technology. This way most of the Governing of the memory less errors can be corrected which are occurred due to capacitor mismatch, limited opamp gain and switch-induced offset errors. the suggested correction techniques feasible to improve the conversion Precesion,conversion speed and lower the power Utilization in an effective wayby relaxing the analog parts when we consider precision matching and high open loop gain.

There is a need of a prototype of a pipelined ADC Errors in preparing and enlarging the digital correction techniques. by an assessment. assuming few errors in a pipelined ADC and these errors can be modelled as a twist in code domain. this depiction is very similar to that distortion in the channels of digital communication system. here we introduce a adaptive linear equalization (LE) for removing 
the unwanted things from signal which shows to be the solution to attain well planned computation. with the help of Linear equalization we can also validate the channel postulation.and the block diagram representation for this error correction is shown in below figure 3 .

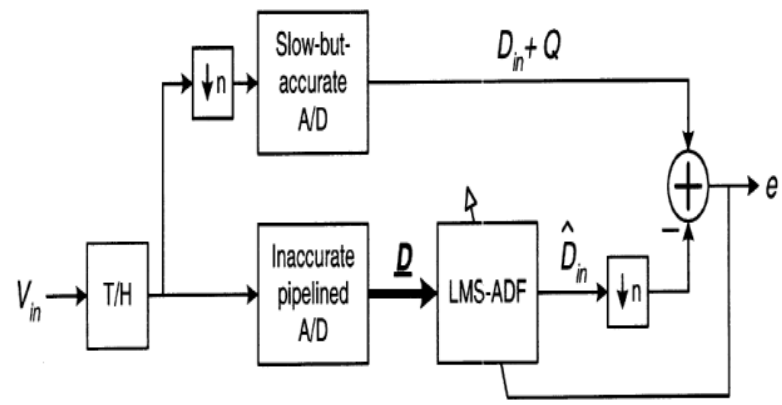

Fig.3Error correction of pipelined ADC by Yun Chiu et.al.

so here the consequences of a particular point errors like capacitor discrepancy, restricted op-amp gain, opamp offset, and sampling key induced offset if these all are not signal dependent then these can be addressed by proposed code domain adaptive FIR filter.here the total procedure is all digital and data driven and fully adjustable and can be operated in background.in this process astrong tradeoff is seen between the accuracy and speed of a pipelined ADCswit the aid of digital correction techniques. So by this way we can translate the analog accuracy issues into the difficult of digital signal processing circuits this proceed towards satisfying the CMOS device scaling when compared to most traditional correction techniques.

Masanori Furuta, Shoji Kawahito, and Daisuke Miyazaki [13]says that the offset errors caused due to charge booser, and gain in accuracies caused by capacitor mismatch,in redundant radix 4 pipelined ADcs will be addressed by digital calibration Technique.

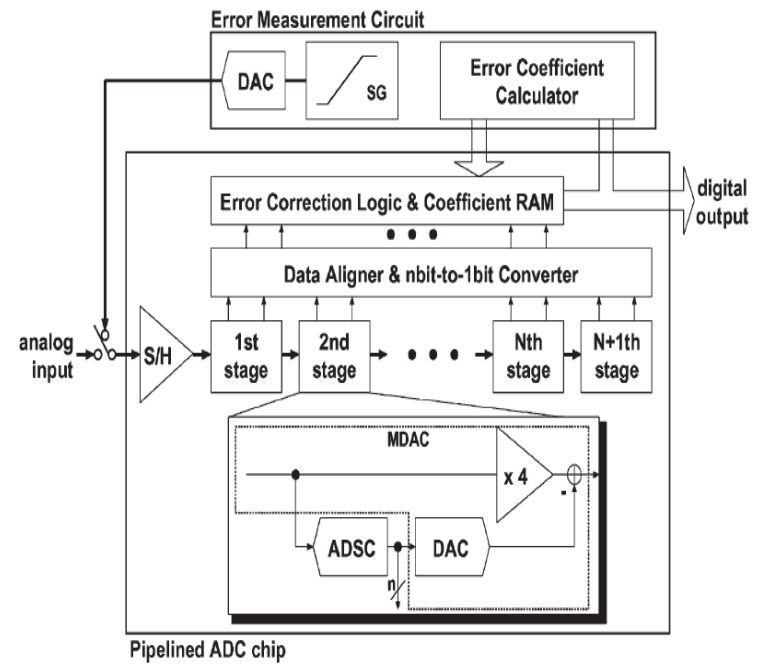

Fig.4Proposed digitally calibrated ADC by Masanori Furutaet.al.

In fig. 4 resolution of each stage is considered to be same for reducing the analysis. the analysis consists of as sample and hold circuit connecting to radix 4 ADC which utilizes the ADSC and DAC with multiplying i.e. MDAC with a residue. The residue is generated from converting and sample and hold circuit with gain 4 . The above discussed analysis proposes a digitally error corrections.

The suggested method makes more comprehensible errorquantifying and eliminate on board circuitry in the ADC. The work station simulation consequences shows that the maximum of 15 LSB of INL in case of a an un calibrated 15-bit ADC is abridged to 0.3 LSB.

A. Tahmasebi, A. Kamali, Z.D. KoozehKanani and J. Sobh [14] proposed analog-to-digital converter with pipelined architecture, deviations from operational amplifier gain and capacitor variations subdue the performance of general pipelined converters. In this paper, internal stage deviations due to gain are addressed with a novel technique. Correction of the error generated in every stage is done in analog domain. The suggested adjustment of plan of action use a distortion free ADC to decide acquired error of the calibration stage. The principle aim of the paper is to make use of profitable, moderate, stunted power, giant resolution $\mathrm{ADC}$ to make gain error free.

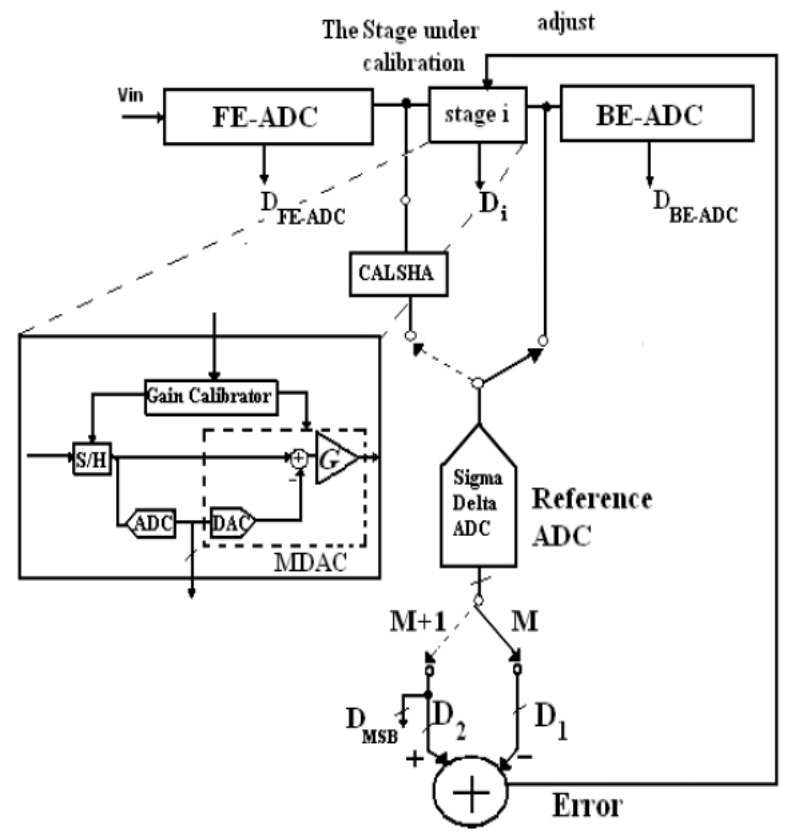

Fig.5Basic idea of calibration algorithm byA.Tahmasebiet.al.

In figure 5 the primary aim beyond the computing scheme mentioned is precise for the inaccuracy in gain of non optimal pipelined stages applied a slow and giant resolution. In a 12 bit pipelined ADC shows that the benefit is calibration accuracy doesn't depend on other stages, as it shows that its SNDR is improved from $39 \mathrm{db}$ to $72 \mathrm{db}$.

Qing Leil, ZhaoHui Wu, Bin Lil and Hailun Wu [15]discusses a digital backdrop error-computation Method for a 16 bit successive approximation pipeline data converter based on LMSA is presented with a 16 bit resolution and ENOB is improved from 10.31 bits to 15.66 bits in this a combination of LMS Algorithm combines with 
a reference $\mathrm{ADC}$ which is a slow but accurate one for calibrating gain error, capacitor discrepancy etc.. the computing algorithm scheme is shown where a LMS algorithm is used with an output vector D of an imprecise pipelined SAR ADC is connected with a LMS adjustable digital FIR Filter.

Andres Amaya, Hector Gomez and ElkimRoa [16] present a method where a fully digital implemental by maintaining the offset accuracy phase calculation approach for offset voltage rectification capability is on slicer output phase by circumvent the input connection to a $\mathrm{CM}$ voltage is developed for high speed correction technique apart from traditional methods.

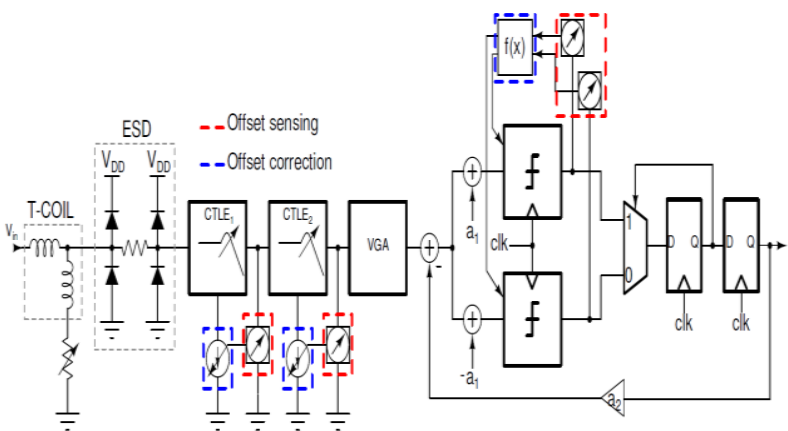

Fig.6 Conventional offset correction by SHS interface by Andres Amaya et.al.

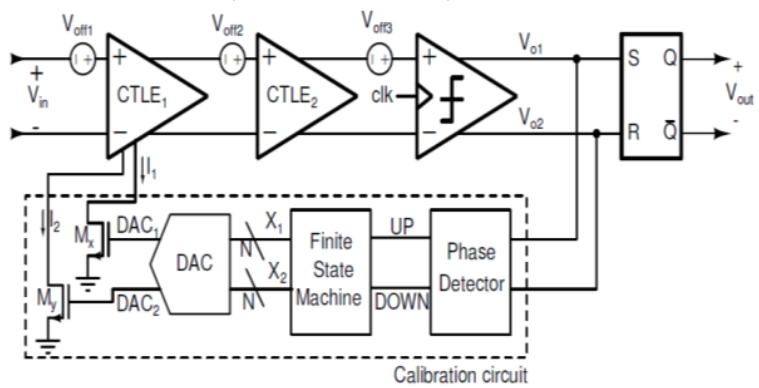

Fig.7Proposed offset compensation by Andres Amaya et.al.

Table1. Comparison of Literature of ADC and Calibration Techniques

\begin{tabular}{|c|c|c|c|c|c|c|}
\hline & [11] & [12] & [13] & [14] & [15] & [16] \\
\hline Type of ADC & $\begin{array}{l}\text { Pipelined Folded } \\
\text { ADC }\end{array}$ & Pipelined ADC & $\begin{array}{l}\text { Radix-4 } \\
\text { pipelined } \\
\text { ADC }\end{array}$ & Pipelined ADC & SAR ADC & $\begin{array}{l}\text { High-speed } \\
\text { digital } \\
\text { interfaces }\end{array}$ \\
\hline $\begin{array}{l}\text { Technique Used } \\
\text { for Calibration }\end{array}$ & $\begin{array}{l}\text { Pipelining and } \\
\text { Background } \\
\text { Offset Trimming }\end{array}$ & $\begin{array}{l}\text { Adaptive digital } \\
\text { technique using } \\
\text { LMS Algorithm }\end{array}$ & $\begin{array}{l}\text { Digital- } \\
\text { computation } \\
\text { ability }\end{array}$ & $\begin{array}{l}\text { Digital- } \\
\text { computation } \\
\text { ability }\end{array}$ & $\begin{array}{l}\text { LMSA for } \\
\text { Digital } \\
\text { backdrop error- } \\
\text { computation } \\
\text { method in SAR } \\
\text { ADC }\end{array}$ & $\begin{array}{l}\text { Offset voltage } \\
\text { correction }\end{array}$ \\
\hline $\begin{array}{l}\text { Errors } \\
\text { Calibrated }\end{array}$ & $\begin{array}{l}\text { Offset of Folding } \\
\text { Amplifier }\end{array}$ & $\begin{array}{l}\text { module errors as } \\
\text { well as capacitor } \\
\text { inequality }\end{array}$ & $\begin{array}{l}\text { Gain errors } \\
\text { and offset } \\
\text { errors }\end{array}$ & $\begin{array}{l}\text { Gain of } \\
\text { operational } \\
\text { amplifier and } \\
\text { mismatch in } \\
\text { capacitors }\end{array}$ & $\begin{array}{l}\text { Capacitor } \\
\text { disparity, gain } \\
\text { error and } \\
\text { reference } \\
\text { voltage offset } \\
\text { error }\end{array}$ & Offset Error \\
\hline
\end{tabular}




\begin{tabular}{|c|c|c|c|c|c|c|}
\hline Technology & $0.5 \mu \mathrm{m}$ & - & - & - & - & $130 \mathrm{~nm}$ \\
\hline INL & $\pm 2.0 \mathrm{LSB}$ & - & $\pm 0.3 \mathrm{LSB}$ & - & - & - \\
\hline$\overline{D N L}$ & \pm 0.5 LSB & - & - & - & - & - \\
\hline ENOB & 13 Bits & - & 15 Bits & 12 Bits & 15.66 & - \\
\hline SNDR & $82 \mathrm{~dB}$ & - & - & $72 \mathrm{~dB}$ & - & - \\
\hline Result & $\begin{array}{l}\text { Background } \\
\text { offset trimming } \\
\text { implemented - } \\
\text { Resolution } \\
\text { improved for } 12 \\
\text { bits }\end{array}$ & $\begin{array}{l}\text { Proposed technique } \\
\text { benefit scaling } \\
\text { CMOS circuit }\end{array}$ & 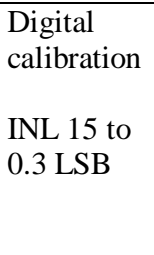 & $\begin{array}{l}\text { SNDR - } 72 \mathrm{~dB} \\
\text { 12-bit ADC. }\end{array}$ & $\begin{array}{l}\text { ENOB } \\
\text { improved to } \\
15.66 \text { bits. }\end{array}$ & Offset decrease \\
\hline
\end{tabular}

\section{CONCLUSION}

This paper focuses on the importance of calibration in ADC and possible sources of errors for improving INL, DNL and ENOB. A detailed study of different state of art is given for reduction in error sources and calibrations for gain and offset errors.

\section{REFERENCES}

1. H S Lee, D A Hodges and P R Gray.: A self-calibrating 15 bit CMOS A/D converter, IEEE Journal of Solid State Circuits, December, 1984.

2. A N Karanicolas, H S Lee and K L Barcrania.: A 15-bit 1Msample/s digitally self-calibrated pipeline ADC, IEEE Journal of Solid State Circuits, December, 1993.

3. H S Lee.: A 12-bit $600 \mathrm{ks} / \mathrm{s}$ digitally self-calibrated pipelined algorithmic ADC, IEEE Journal of Solid State Circuits, April, 1994.

4. T H Shu, B S Song and K. Bacrania.: A 13-bit 10-Msample/s ADC digitally calibrated with oversampling delta-sigma converter, IEEE Journal of Solid State Circuits, April, 1995.

5. U K Moon and B S Song.: Background digital calibration techniques for pipelined ADCS, IEEE Transactions and Circuit Systems-II, February, 1997.

6. S U Kwak, B S Song and K Bacrania.: A 15-b, 5-Msample/s low-spurious CMOS ADC, IEEE Journal of Solid State Circuits, December, 1997.

7. J M Ingino and B A Wooley.: A continuously calibrated 12-b, 10-MS/s, 3.3-V A/D converter, IEEE Journal of Solid State Circuits, December, 1998.

8. O E Erdogan, P J Hurst and S H Lewis.: A 12-bit digitalbackground-calibrated algorithmic ADC with 90-dB THD, IEEE Journal of Solid State Circuits, December, 1999.

9. S Sonkusale, J Van Der Spiegel and K Nagaraj.: True background calibration technique for pipelined ADC, IEEE Electronic Letter, April, 2000.

10. J Ming and S H Lewis.: An 8-bit 80-Msample/s pipelined analog to digital converter with background calibration, IEEE Journal of Solid State Circuits, October, 2001.

11. M J Choe and B S Song and K Bacrania.: A 13-b 40MSamples/s CMOS Pipelined Folding ADC with Background Offset Trimming, IEEE Journal of Solid State Circuits, December 2000.

12. Y Chiu, C W Tsang, B Nikolic and P R Gray.: Least Mean Square Adaptive Digital Background Calibration of Pipelined Analog-to-Digital Converters, IEEE Transactions and Circuit Systems-I, January, 2004.

13. M Furuta, S Kawahito and D Miyazaki.: A Digital-Calibration Technique for RedundantRadix-4 Pipelined Analog-to-Digital Converters, IEEE Transactions on Instrumentation and Measurement, December, 2007.
14. A Tahmasebi, A Kamali, Z D Koozeh Kanani and J Sobh.: A Simple Background Inter-stage Gain Calibration Technique for Pipeline ADCs, ICSAP, 2009.

15. Q Leil, Z H Wu, B Lil and Hailun Wu.: Digital Background Calibration for Pipelined SAR ADC Based On LMS algorithm, International IEEE Conference of EDSC, 2013.

16. A Amaya, H Gomez and E Roa.: A Digital Offset Correction Method for High SpeedAnalog Front-Ends, SBCCI, 2016 